\title{
Bryaxis nouveaux ou méconnus du Nord de l'Italie (Coleoptera: Pselaphidae)
}

\author{
par
}

\author{
Claude BESUCHET *
}

Avec 21 figures

\section{AbStract}

New of badly known Bryaxis from Northern Italy (Coleoptera: Pselaphidae).-In this paper a list is given of the 51 species and 12 subspecies of the genus Bryaxis Kug. known from Northern Italy, 3 new species (Bryaxis frustratus n. sp., effeminatus n. $\mathrm{sp}$. and mirificus n. sp.) and 2 new subspecies (Bryaxis halbherri pacei $\mathrm{n}$. ssp. and longulus inflatus n. ssp.) are described, the synonymy of one taxon is discussed, new characters and localities are added for 2 other species.

Le genre Bryaxis Kug., dont l'aire de répartition comprend toute la région paléarctique, est très richement représenté dans le Nord de l'Italie, c'est-à-dire dans tout le territoire occupé par les Alpes, des Alpes Maritimes aux Alpes Juliennes. En effet, 51 espèces et 12 sous-espèces sont actuellement connues de cette région, compte tenu des taxa décrits dans ce travail et dans deux publications actuellement sous presse de M. H. Daffner.

Ces Bryaxis du Nord de l'Italie sont non seulement très nombreux, mais aussi fort variés. Certaines espèces sont largement répandues; d'autres au contraire sont étroitement localisées. Elles vivent suivant les cas dans les zones humides, dans la litière des forêts ou sous les pierres en altitude, mais aussi profondément enfoncées dans les sols forestiers ou dans les grottes. Je donne ci-dessous la liste de ces 51 Bryaxis, dans l'ordre alphabétique des noms valides, en citant également les sous-espèces connues du Nord de l'Italie et les synonymes, parmi lesquels figurent aussi les noms donnés jadis aux variations poecilandriques.

* Muséum d'Histoire naturelle, case postale 434, CH-1211 Genève 6, Suisse.

Rev. Suisse De Zool., T. 90, 1983 
Bryaxis alpestris Dod.; argus Kr.; bergamascus Stolz (b. bergamascus Stolz et b. sorinensis Stolz = breiti Bes.); bericus Pace; brachati Bes.; bulbifer Reichb. (= extremitalis Reitt.); carinula Rey (= punctipennis Rey); cateniger Krauss (c. cateniger Krauss $=$ gspani Kar., $c$. anabates Holdh. et $c$. bothrophorus Stolz); chevrolati Aubé (= mirabilis Kar.); collaris Baudi (= manueli Sharp = germanus Reitt. = foemineus Fiori); colledanii Daffner; curtisi Leach (c. curtisi Leach et $c$. orientalis Kar.); effeminatus n. sp.; emilianus Stolz; focarilei Bes.; frustratus n. sp.; gallicus Reitt. (= gruardeti Caillol = fortipes Dod. = apuanus Rasetti); ganglbaueri Stolz; glabricollis Schm. (g. glabricollis Schm. = guranyii Csiki et $g$. simplex Baudi $=$ dudichi Reitt.); grouvellei Reitt. (= sculpticollis Reitt.); halbherri Reitt. (h. hablherri Reitt. et h. pacei n. ssp.); italicus Baudi; judicariensis Dod. (= scherleri Bes.); kahleni Pace; konecznii Mach.; kruegeri Mach. (= machulkai Bes. = hoelzeli Kar. = kahleri Hölzel = simplicicornis Bes. $)$; lagari Halbh. (= lictor Flach = blattnyorum Reitt.); latebrosus Reitt. (= ravouxi Grilat $=$ schneideri Reitt.); lessinicus Pace; liguricus Dod.; longulus Kiesw. (l. longulus Kiesw. et $l$. inflatus n. ssp.); mirificus n. sp.; monguzzii Bes.; muscorum Kiesw.; nodicornis Aubé (n. nodicornis Aubé); oertzeni Reitt. (= rosai Bin.); pavani Tam. (= boldorii Bin.); pedemontanus Bes.; pentagonoceras Stolz; persicoi Rasetti; picteti Tourn. ( $p$. picteti Tourn. = marthae Reitt. = alpinus $\mathrm{Rey}=$ atavicus Fiori = incertulus Mach.); pinkeri Stolz; porsenna Reitt. (= heteromorphus Fiori = mimus Dod. = ticinensis Bes.); procerus Gredl. (= noesskei Ganglb. = brasavolai Tam.); puncticollis Denny (= validus Aubé $=$ gracilipes Raffr.); reissi Mach.; rugosicollis Fiori (r. rugosicollis Fiori $=$ muliebris Künn., r. montellensis Megg., $r$. peresinottoi Megg. et $r$. cadamuroi Megg.); sculpticornis Guillb. (= alticola Dod. = woerndlei Holdh.); trigonoceras Holdh.; troglodytes Fiori ( $t$. troglodytes Fiori, $t$. pierottii Bes. et $t$. lansbergeri Daffner); vicinus Dod.

C'est grâce à l'obligeance de MM. K. Burmann, Innsbruck, A. Focarile, St. PierreAoste, J. Jelinek, Prague, R. Monguzzi, Milan ,R. Pace, Monteforte et H. Schönmann, Vienne, que j'ai pu rédiger ce travail; je les en remercie, bien sincèrement.

\section{Bryaxis frustratus $\mathrm{n}$. sp.}

Long. $1,7 \mathrm{~mm}$. Coloration entièrement d'un brun rougeâtre peu foncé; pubescence de la face dorsale simple, formée de soies de longueur moyenne. Tête $(0,30 / 0,33 \mathrm{~mm})$ légèrement plus large que longue, nettement moins large que le pronotum. Lobe frontal de largeur moyenne $(0,16 \mathrm{~mm})$, court et transverse, légèrement élargi d'avant en arrière; bord antérieur nettement anguleux; moitié postérieure distinctement chagrinée; dépression frontale bien marquée. Fossettes interoculaires petites, profondes, simples. Vertex avec une carénule médiane entière, égale sur toute sa longueur mais située en arrière dans une dépression assez bien marquée. Tempes arrondies. Bord antérieur de l'épistome prolongé de chaque côté jusqu'à l'oeil par une carénule bien distincte en vue dorsale. Palpes maxillaires relativement courts; $2^{\mathrm{e}}$ article un peu courbé, nettement élargi dans la partie apicale, celle-ci avec quelques petits tubercules, surtout sur la face ventrale; $3^{\mathrm{e}}$ article avec aussi quelques petits tubercules; dernier article $(0,22 / 0,08 \mathrm{~mm})$ robuste, sa massue presque deux fois et demie plus longue que large, la plus grande largeur située au quart basal. Antennes relativement courtes $(0,62 \mathrm{~mm})$; article 3 nettement plus long que large, 4 presque aussi long que large, 5, 6 et 7 nettement plus larges que longs, 8 transverse, 9 nettement plus large que les articles du funicule, transverse, 10 encore distinctement plus large, transverse également, $11(0,14 / 0,09 \mathrm{~mm})$ un peu plus long que les quatre articles précédents réunis. Pronotum $(0,42 / 0,43 \mathrm{~mm})$ presque aussi long que large; côtés normalement arrondis et nettement atténués en arrière; disque 
convexe, non ponctué. Elytres réunis $(0,68 / 0,68 \mathrm{~mm})$ aussi larges que longs, leur ponctuation superficielle; callosité humérale arrondie, bien marquée; fossettes basales très profondes, égales; dépression dorsale marquée presque jusqu'au tiers antérieur de l'élytre; fossette marginale peu distincte en vue dorsale. Pattes moyennement longues (tibias III : 0,46 mm; tarses III : 0,34 mm).

Caractères sexuels du mâle. Yeux relativement bien développés, saillants, formés chacun d'une vingtaine d'ommatidies. Scape (fig. 1) $(0,12 / 0,075 \mathrm{~mm})$ court, légèrement
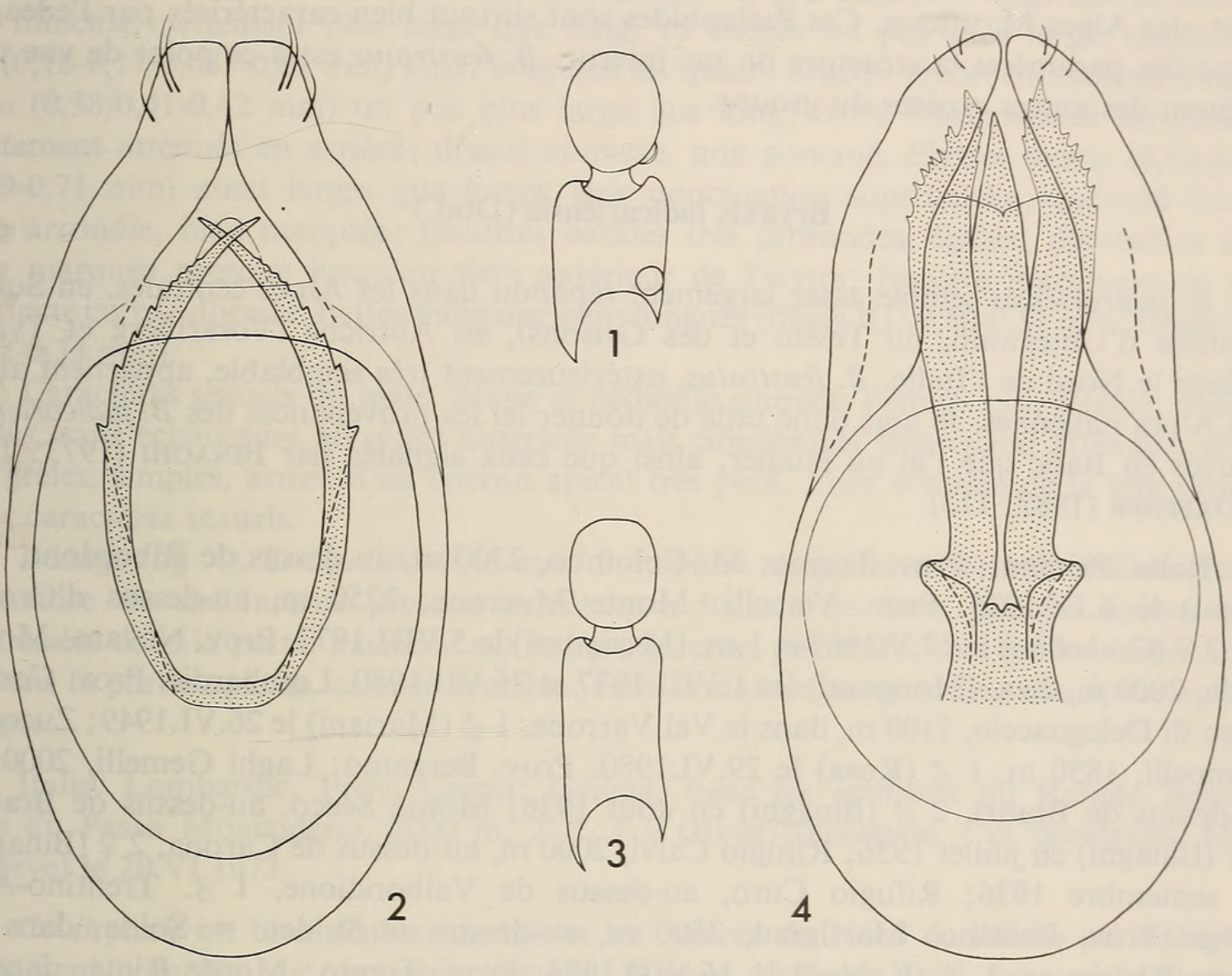

FIG. 1 à 4 .

Bryaxis Kug. 1: B. frustratus n. sp., scape et pédicelle du mâle, face dorsale; 2 : id., édéage, face dorsale; $3: B$. effeminatus n. sp., scape et pédicelle du mâle, face dorsale; 4: id., édéage, face dorsale.

et régulièrement élargi de la base à l'apex, orné sur la partie basale interne de la face dorsale d'une petite fossette tomenteuse. Pédicelle $(0,075 / 0,06 \mathrm{~mm})$ un peu plus long que large, distinctement moins large que le scape, ovalaire, orné sur la base du bord inférieur d'une petite saillie arrondie, légèrement rebordée. Fémurs très renflés (forma inflatipes) (largeur fémurs I et III : 0,15 et $0,18 \mathrm{~mm}$ ); tibias I relativement robustes, ornés d'une échancrure profonde au quart antérieur; tibias III robustes, le tiers postérieur du bord interne distinctement atténué et orné d'une frange de soies, l'apex armé d'un petit éperon. Face ventrale de la tête ornée d'une dépression transverse profonde, limitée en avant de chaque côté par une dent arrondie assez élevée. 
Edéage (fig. 2). Long. $0,41 \mathrm{~mm}$. Paramères robustes, tronqués à l'apex. Armature du sac interne symétrique, formée de deux longues épines grêles, atténuées en pointe à l'extrémité, dentelées sur une partie du bord externe.

Italie. Lombardie. Prov. Brescia: Cima Tombea, 1 ô œdimère (Pechlaner) (holotype, Mus. Prague) trouvé le 13.VI.1935. Cet exemplaire est resté dans la collection Machulka avec l'identification «ad oreophilus ».

Ce nouveau Bryaxis appartient au groupe d'oreophilus, qui compte maintenant cinq espèces: oreophilus Meixner de Styrie, witzgalli Daffner (1982: 113) de Carinthie, judicariensis Dod. des Alpes centrales, frustratus n. sp. des Alpes Brescianes et gallicus Reitt. des Alpes Maritimes. Ces Psélaphides sont surtout bien caractérisés par l'édéage: forme des paramères et structure du sac interne. $B$. frustratus est à ce point de vue très différent des autres espèces du groupe.

\section{Bryaxis judicariensis (Dod.)}

B. judicariensis semble assez largement répandu dans les Alpes centrales, en Suisse (cantons d'Unterwald, du Tessin et des Grisons), en Autriche (Vorarlberg et Tyrol) et dans le Nord de l'Italie. $B$. frustratus, extérieurement très semblable, appartient aussi aux Alpes italiennes. Je juge donc utile de donner ici les provenances des B. judicariensis trouvés en Italie que j'ai pu étudier, ainsi que ceux signalés par BINAGHI (1973: 117) et DAFFNER (1982: 113).

Italie. Piémont. Prov. Torino: M. Colombo, 2300 m, au-dessus de Ribordone, 1 đ (Olmi) le 6.IX.1976. Prov. Vercelli: Monte Mucrone, $2250 \mathrm{~m}$, au-dessus d'Oropa, 2 o 3 ㅇ (Comellini) le 27.V.1967 et 1 ex. (Monguzzi) le 5.VIII.1977. Prov. Novara: Monte Zeda, 2000 m, 2 ex. (Monguzzi) les 1.VIII.1977 et 26.VII.1980. Lombardie. Prov. Como: Lago di Deleguaccio, 2100 m, dans le Val Varrone, 1 ô (Mariani) le 26.VI.1949; Zuccone Campelli, 1850 m, 1 ơ (Rosa) le 29.VI.1980. Prov. Bergamo: Laghi Gemelli, 2000 m, au-dessus de Branzi, 2 đิ (Binaghi) en août 1936; Monte Secco, au-dessus de Branzi, 1 ô (Binaghi) en juillet 1936; Rifugio Calvi, 2000 m, au-dessus de Carona, 2 \& (Binaghi) en septembre 1936; Rifugio Curò, au-dessus de Valbondione, 1 đ.. Trentino-Alto Adige. Prov. Bolzano: Martlgrad, $2500 \mathrm{~m}$, au-dessus de Sulden = Solda, dans les Alpes Rhétiques, 1 ơ (Kahlen) le 16.VIII.1974. Prov. Trento: Monte Rima, dans la Giudicarie, 1 đิ 1 ㅇ (Mancini) (types, Coll. Dodero, Gênes) en août 1917; Cimon Rava, au-dessus de Bieno, 1 ô 3 ㅇ (Binaghi) en juillet 1933.

\section{Bryaxis effeminatus $\mathrm{n}$. $\mathrm{sp}$.}

Long. 1,75-1,8 mm. Coloration entièrement d'un brun rougeâtre clair ou peu foncé; pubescence de la face dorsale simple, formée de soies de longueur moyenne. Tête $(0,30 / 0,33 \mathrm{~mm})$ légèrement plus large que longue, nettement moins large que le pronotum. Lobe frontal relativement large $(0,19 \mathrm{~mm})$, court et transverse, subparallèle; bord antérieur nettement anguleux; partie postérieure un peu chagrinée; dépression frontale bien marquée, large et peu profonde. Fossettes interoculaires petites, profondes, simples. Vertex avec une carénule médiane entière, égale sur toute sa longueur mais située en arrière dans une dépression assez bien marquée. Tempes arrondies. Yeux peu saillants, formés chacun dans les deux sexes d'une dizaine d'ommatidies. Bord antérieur de l'épistome prolongé de chaque côté jusqu'à l'œil par une carénule peu distincte 
en vue dorsale. Palpes maxillaires normalement développés; $2^{\mathrm{e}}$ article un peu courbé, nettement élargi dans la partie apicale, celle-ci avec quelques petits tubercules localisés sur la face ventrale; $3^{\mathrm{e}}$ article avec aussi quelques petits tubercules; dernier article $(0,26-$ $0,28 / 0,09 \mathrm{~mm}$ ) robuste, sa massue légèrement plus de deux fois et demie plus longue que large, la plus grande largeur située au quart basal. Antennes de longueur moyenne $(0,68-0,69 \mathrm{~mm})$, semblables dans les deux sexes; scape (fig. 3) $(0,15 / 0,065 \mathrm{~mm})$ subcylindrique, simple, un peu plus de deux fois plus long que large; pédicelle $(0,07 / 0,055-0,06 \mathrm{~mm})$ simple, un peu plus long que large, légèrement moins large que le scape; article 3 nettement plus long que large, 4 et 5 un peu plus longs que larges, 6 et 7 aussi longs ou à peine plus longs que larges, 8 légèrement plus large que long, 9 bien plus làrge que les articles du funicule, nettement plus large que long, 10 encore un peu plus large, transverse, $11(0,16-0,17 / 0,085-0,09 \mathrm{~mm})$ aussi long que les quatre articles précédents réunis. Pronotum $(0,38 / 0,41-0,42 \mathrm{~mm})$ un peu plus large que long; côtés normalement arrondis et nettement atténués en arrière; disque convexe, non ponctué. Elytres réunis $(0,68-0,71 /$ 0,69-0,71 $\mathrm{mm}$ ) aussi larges que longs, leur ponctuation superficielle; callosité humérale arrondie, bien marquée; fossettes basales très profondes, égales; dépression dorsale marquée presque jusqu'au tiers antérieur de l'élytre; fossette marginale un peu distincte en vue dorsale. Pattes moyennement longues (tibias III : 0,50-0,51 mm; tarses III: $0,32-0,33 \mathrm{~mm})$.

Caractères sexuels du mâle. Scape et pédicelle simples. Fémurs non renflés; tibias I grêles, sans échancrure au quart antérieur mais simplement légèrement atténués; tibias III grêles, simples, armés d'un éperon apical très petit. Face ventrale de la tête simple, sans caractères sexuels.

Edéage (fig. 4). Long. 0,43-0,46 mm. Paramères simplement arrondis à l'extrémité. Armature du sac interne presque symétrique, formée de deux grandes épines bien chitinisées dans la partie basale et sur le bord interne, peu chitinisées sur le bord externe et dans la partie apicale, celle-ci dentelée; cette armature porte encore une épine apicale simple.

Italie. Lombardie. Prov. Como: Grigna, dans les environs du Refuge Bogani, près du Passo Moncodeno, $2000 \mathrm{~m}, 2 \hat{\sigma}, 1$ ㅇ (Rosa) (holotype $\hat{o}$ et paratypes, Mus. Genève) le 29.VI.1973.

Ce Bryaxis est bien distinct de toutes les espèces du Nord de l'Italie par l'absence quasiment complète de caractères sexuels. Il était d'ailleurs resté dans ma collection depuis des années parmi les femelles non identifiables!

\section{Bryaxis mirificus n. sp.}

Long 2,0 mm. Coloration entièrement d'un brun rougeâtre peu foncé; pubescence de la face dorsale simple, formée de soies de longueur moyenne. Tête $(0,40 / 0,40 \mathrm{~mm})$ aussi longue que large, un peu moins large que le pronotum. Lobe frontal étroit $(0,16 \mathrm{~mm})$, légèrement plus long que large, subparallèle; bord antérieur un peu anguleux au milieu; dépression frontale particulièrement petite, étroite $(0,04 \mathrm{~mm})$ mais profonde, marquée seulement dans la moitié antérieure du lobe frontal; moitié postérieure de celui-ci ornée d'une carène médiane saillante, assez élevée, prolongée en arrière jusque sur le bord postérieur de la tête mais en étant cependant un peu moins haute. Fossettes interoculaires petites, profondes, prolongées chacune en avant par une dépression bien marquée quoique peu profonde, convergeant vers la dépression frontale. Tempes un peu anguleuses et saillantes en arrière des yeux. Bord antérieur de l'épistome prolongé de chaque côté 
jusqu'à l'œil par une carénule bien distincte en vue dorsale. Palpes maxillaires assez grands; $2^{\mathrm{e}}$ article nettement élargi de la base à l'apex, un peu courbé, sans tubercules; $3^{\mathrm{e}}$ article orné de quelques tubercules superficiels sur la face dorsale et sur la face ventrale; dernier article $(0,32 / 0,14 \mathrm{~mm})$ robuste, sa massue deux fois plus longue que large, la plus grande largeur située au quart basal. Antennes de longueur moyenne $(0,96 \mathrm{~mm})$;
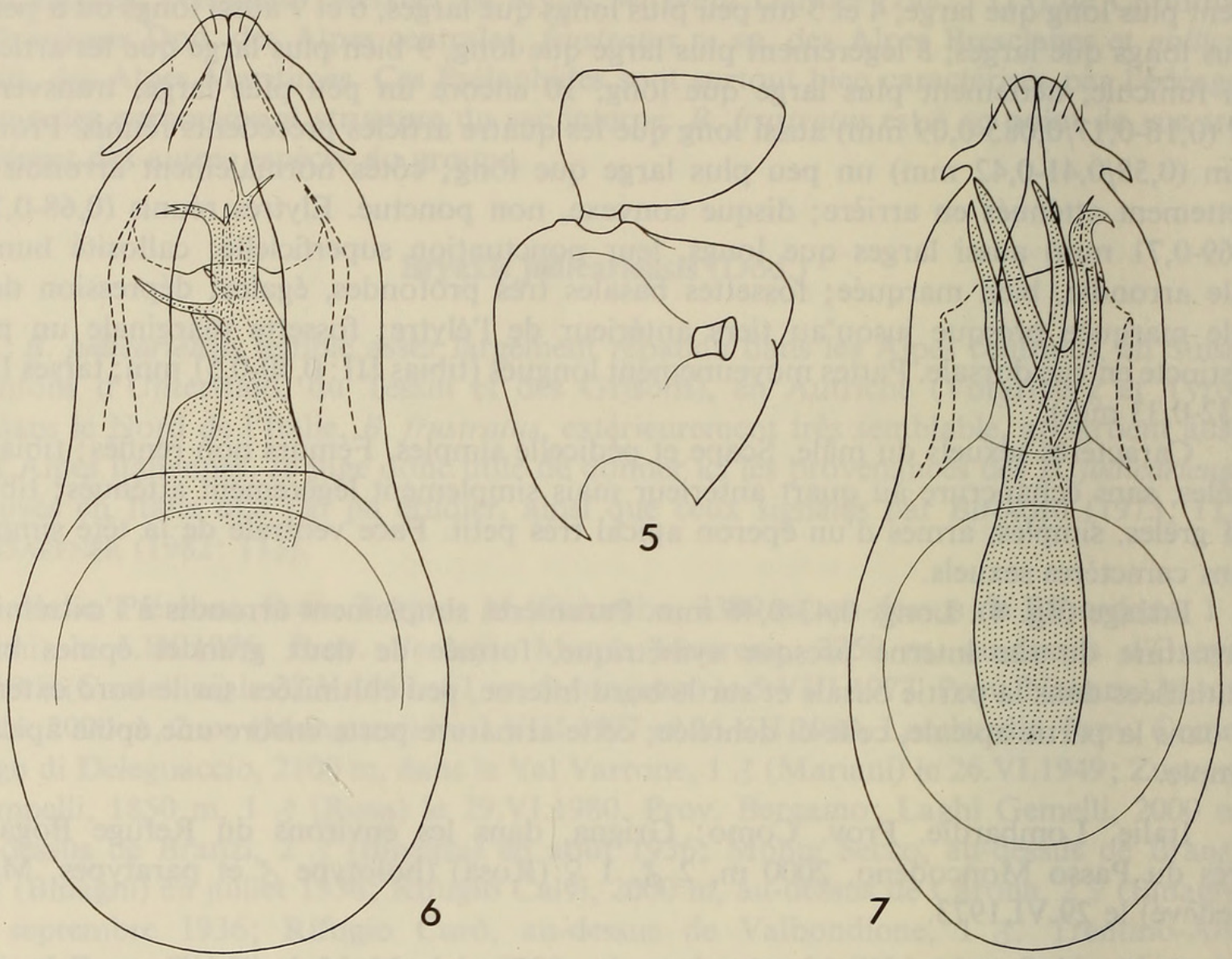

FIG. 5 à 7.

Bryaxis Kug. 5: B. mirificus n. sp., scape et pédicelle du mâle, face dorsale; 6: id., édéage, face dorsale; 7: B. emilianus Stolz, édéage, face dorsale.

articles 3, 4 et 5 nettement plus longs que larges, 6 et 7 globuleux, aussi longs que larges, 8 nettement plus large que long, 9 bien plus large que les articles du funicule, aussi long que large, 10 encore un peu plus large, un peu plus large que long, $11(0,17 / 0,10 \mathrm{~mm})$ aussi long que les trois articles précédents réunis. Pronotum $(0,45 / 0,46 \mathrm{~mm})$ à peine plus large que long; côtés normalement arrondis et nettement atténués en arrière; disque convexe, non ponctué. Elytres réunis $(0,76 / 0,76 \mathrm{~mm})$ aussi larges que longs, leur ponctuation presque complètement effacée; callosité humérale atrophiée; fossettes basales très profondes, égales; dépression dorsale courte, marquée jusqu'au cinquième antérieur de l'élytre; fossette marginale un peu distincte en vue dorsale. Pattes relativement longues (tibias III : $0,65 \mathrm{~mm}$ ), les tarses longs et grêles (tarses III: $0,51 \mathrm{~mm}$ ). 
Caractères sexuels du mâle. Yeux peu développés, assez saillants cependant, formés chacun de 9 ommatidies. Scape (fig. 5) $(0,22 / 0,20 \mathrm{~mm})$ très robuste, presque aussi large que long, orné sur la face dorsale d'un petit tubercule grêle saillant; face interne prolongée, du côté ventral, par un lobe arrondi assez convexe. Pédicelle $(0,095 / 0,145 \mathrm{~mm})$ prolongé sur son bord interne par un lobe arrondi assez épais, non rebordé. Fémurs non renflés; tibias I grêles, ornés d'une petite échancrure au quart antérieur; tibias III simples, de la même largeur sur presque toute la longueur, armés d'un petit éperon apical. Face ventrale de la tête avec une dépression transverse elliptique, relativement profonde, limitée en avant, de chaque côté, par une petite dent plus large que haute et prolongée par deux ou trois soies.

Edéage (fig. 6). Long. 0,48 mm. Paramères armés un peu avant l'apex d'une dent latérale grêle assez longue.Armature du sac interne asymétrique, complexe, avec une dent basale assez robuste et des épines grêles en position apicale et médiane.

Italie. Lombardie. Prov. Bergamo: Valpiana, M. Alben, 1000 m, 1 ๙ (Monguzzi) (holotype, Coll. R. Monguzzi) le 25.IV.1980 sous une pierre profondément enfoncée dans la forêt de hêtres et sapins.

Cette très belle espèce est nettement apparentée à $B$. emilianus Stolz, qui se trouve d'ailleurs aussi à Valpiana; elle en diffère par tout un ensemble de caractères: bord antérieur du lobe frontal faiblement anguleux; dépression frontale nettement plus courte; carène médiane de la tête plus longue et plus saillante; antennes distinctement moins allongées; scape du mâle plus large et plus convexe sur sa face interne; pédicelle du mâle plus développé transversalement; enfin édéage bien différent (cf. fig. 6 et 7), tant par les paramères que par l'armature du sac interne.

\section{Bryaxis halbherri (Reitt.)}

ReITTER (1885: 368) a décrit l'exemplaire trouvé par Halbherr comme étant un mâle; Ganglbauer (1895: 834) a déjà été moins catégorique dans sa description. C'est en fait une femelle. C'est Dodero (1900: 412) qui a découvert et fait connaître le mâle de ce Bryaxis.

MM. R. Pace et R. Monguzzi ont récolté chacun un mâle d'un Bryaxis un peu différent de halbherri, qui appartient à mon avis à une sous-espèce de celui-ci.

B. halbherri halbherri (Reitt.). Long. 1,7-1,8 mm. Antennes (long. 0,76-0,80 mm) de longueur moyenne; articles 4 et 5 à peine plus longs ou aussi longs que larges, 6 et 7 légèrement ou un peu plus larges que longs, 8,9 et 10 transverses. Pronotum $(0,39-0,41 /$ $0,42-0,44 \mathrm{~mm}$ ) légèrement plus large que long.

Caractères sexuels du mâle. Tête $(0,32-0,33 / 0,33-0,35 \mathrm{~mm})$ à peine plus large que longue, les yeux étant relativement saillants, formés chacun de 9 à 12 ommatidies. Dernier article des palpes maxillaires $(0,29-0,31 / 0,09-0,105 \mathrm{~mm})$ de taille moyenne. Scape (fig. 8) $(0,17-0,19 / 0,085-0,10 \mathrm{~mm})$ déprimé, nettement et régulièrement élargi de la base jusque dans la région apicale, l'angle apical interne assez saillant mais arrondi, orné d'un petit tubercule très superficiel, plus ou moins distinct; pédicelle bien moins large que le scape, un peu plus long que large, ovalaire, simple. Fémurs un peu renflés; tibias I ornés d'une échancrure profonde au quart antérieur; tibias III (long. 0,46-0,49 mm) un peu élargis de la base jusqu'au quart postérieur, le bord interne caréné et légèrement dentelé entre le milieu et le quart postérieur, nettement échancré dans la partie apicale et armé à l'extrémité d'un petit éperon robuste. 
Caractères sexuels de la femelle. Tête $(0,32 / 0,31-0,32 \mathrm{~mm})$ pratiquement aussi longue que large, les yeux étant réduits chacun à deux ou trois ommatidies dépigmentées. Dernier article des palpes maxillaires $(0,33-0,35 / 0,12 \mathrm{~mm})$ assez grand. Scape $(0,18 /$ 0,075-0,08 $\mathrm{mm}$ ) déprimé, subparallèle, simple.

Edéage (fig. 9). Long. 0,36-0,40 mm. Paramères de longueur moyenne, un peu élargis au milieu. Armature du sac interne un peu asymétrique, formée de deux lames
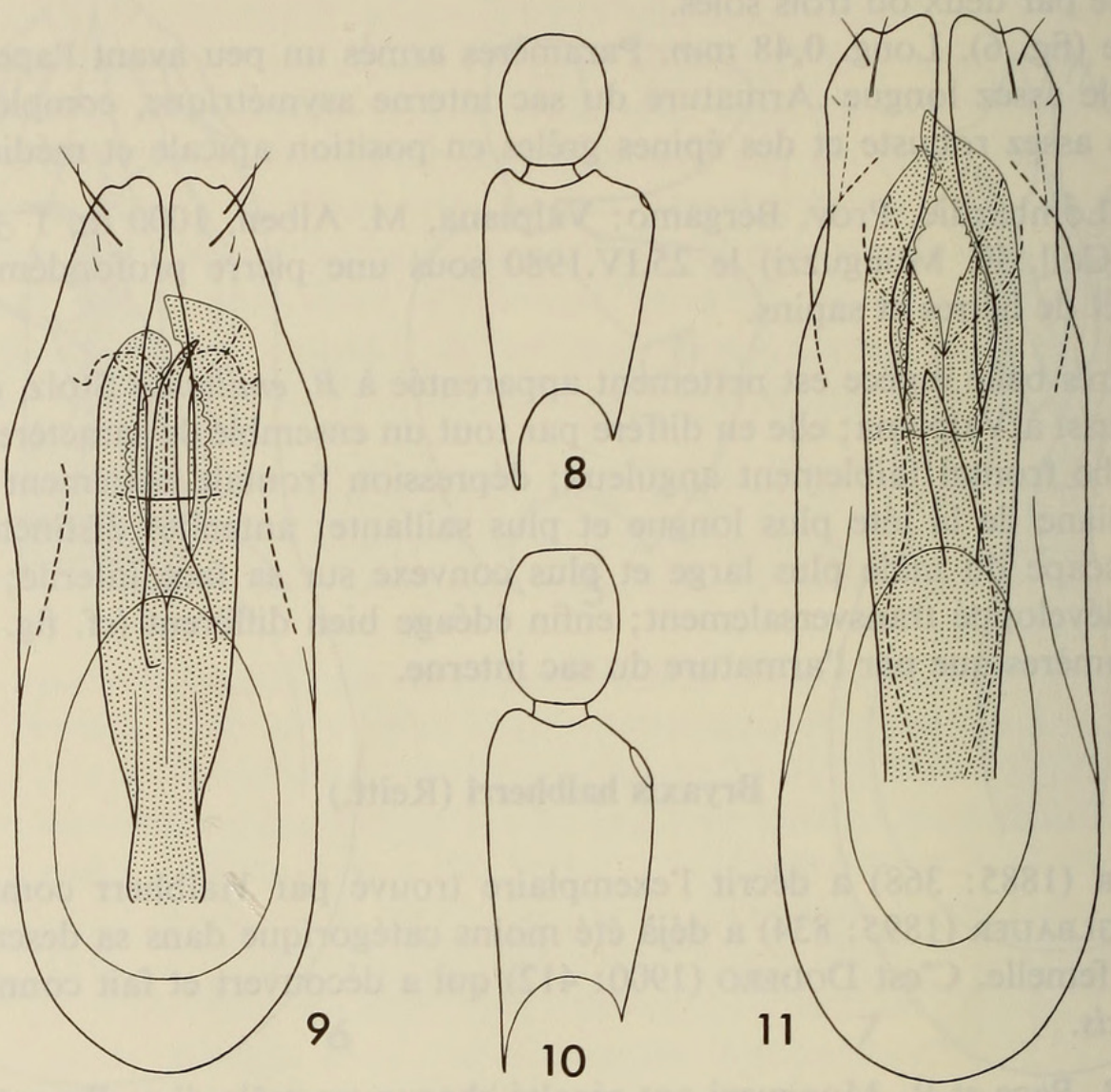

FIG. 8 à 11.

Bryaxis Kug. 8: B. halbherri halbherri Reitt., scape et pédicelle du mâle, face dorsale; 9: id., édéage d'un exemplaire de Pian delle Fugazze, face dorsale; 10: B. halbherri pacei $\mathrm{n}$. ssp., scape et pédicelle du mâle, face dorsale; 11: id., édéage de l'exemplaire du Monte Baldo, face dorsale.

allongées repliées sur elles-mêmes, le bord interne supérieur dentelé; cette armature porte encore une épine apicale simple, plus ou moins développée, particulièrement grande chez les mâles de la région de Vallarsa (fig. 9).

Italie. Trentino-Alto Adige. Prov. Trento: Cima Mandriolo, 1 우 (von Friedl); Lavarone, 1 đิ 1 ㅇ (Dodero) le 20.VIII.1899; Carbonare près de Folgaria, 1 ô (Strupi) le 30.V.1935; Serrada, 1 ô (Pechlaner) le 15.VI.1936 sous des pierres; Vallarsa, 2 o (von Friedl); Pian delle Fugazze, au-dessus de Vallarsa, 1 ㅇ (Halbherr) (type, Mus. Paris) en juillet 1883 dans un tamisage de litière de hêtre à $1200 \mathrm{~m}$; Pian delle Fugazze, 2 ô (von Friedl) et 1 ô (Pace) le 15.VI.1973 à 1000 m. 
B. halbherri pacei n. sp. Long. 1,95 mm. Antennes (long. 0,88 mm) assez longues; articles 4 et 5 un peu plus longs que larges, 6 et 7 à peine plus longs que larges, 8 aussi large que long, 9 et 10 un peu plus larges que longs, $11(0,19 / 0,10 \mathrm{~mm})$ aussi long que les quatre articles précédents réunis. Pronotum $(0,43 / 0,46-0,48 \mathrm{~mm})$ un peu plus large que long.

Caractères sexuels du mâle. Tête $(0,35 / 0,32-0,33 \mathrm{~mm})$ légèrement plus longue que large, les yeux étant peu saillants, formés chacun de 5 à 8 ommatidies. Dernier article des palpes maxillaires (0,35-0,36/0,10-0,105 mm) assez grand. Scape (fig. 10) $(0,21 / 0,09-$ $0,095 \mathrm{~mm}$ ) déprimé, un peu élargi de la base jusqu'au quart apical du bord interne, orné un peu en avant de celui-ci d'un tubercule aplati; pédicelle nettement moins large que le scape, un peu plus long que large, ovalaire, simple. Fémurs nettement renflés (largeur des fémurs I, II et III: $0,15,0,14$ et $0,18 \mathrm{~mm}$ ); tibias I ornés d'une échancrure profonde au quart antérieur; tibias III (long. 0,56-0,57 mm) robustes, un peu élargis de la base jusqu'au quart postérieur, le bord interne caréné et distinctement dentelé entre le milieu et le quart postérieur, nettement échancré dans la partie apicale et armé à l'extrémité d'un petit éperon robuste.

Edéage (fig. 11). Long. $0,46 \mathrm{~mm}$. Paramères particulièrement longs, subparallèles. Armature du sac interne un peu asymétrique, formée de deux lames allongées repliées sur elles-mêmes, le bord interne supérieur dentelé, le bord interne inférieur avec une ou deux dents minuscules; cette armature porte encore, dans sa partie médiane, une épine simple relativement grande.

Italie. Trentino-Alto Adige. Prov. Trento: Monte Baldo, Bocca di Navene, au fond de la vallée, 1340 m, 1 đ (Pace) (holotype, Mus. Genève) le 15.VI.1972 dans un lavage de terre dans la hêtraie. Lombardie. Prov. Brescia: Piani di Vaghezza, 1300 m, dans le Val Trompia, 1 ơ (Monguzzi) (paratype, Coll. R. Monguzzi) le 31.V.1981 dans la terre prélevée sous des pierres profondément enfoncées, dans la forêt de hêtres, sapins et ormes.

Cette sous-espèce est dédiée à M. R. Pace, de Monteforte, qui l'a découverte et qui m'a généreusement donné l'holotype. Les deux mâles connus de ce Bryaxis halbherri pacei sont rigoureusement identiques.

\section{Bryaxis longulus (Kiesw.)}

Bryaxis longulus Kiesenwetter [in KüSTER 1849: 98, type đ̃: Oberkrain/Yougoslavie (! Mus. Munich)] est assez fréquent en Croatie, Slovénie, Carinthie, Styrie, Frioul et Vénétie; il existe également dans la partie orientale de la Lombardie, où BINAGHI (1973: 133) l'a signalé de quatre localités de la province de Brescia.

Les exemplaires du Piémont appartiennent à une race distincte nommée inflatus par Machulka, mais restée in litteris. La capture d'une belle série d'exemplaires par le $\mathrm{D}^{\mathrm{r}} \mathrm{A}$. Focarile me permet de confirmer la validité de cette race géographique et de la décrire.

B. longulus longulus (Kiesw.). Carénule médiane du vertex ornée en avant d'une petite dent saillante. Scape du mâle (fig. 12) nettement renflé (largeur: 0,08-0,09 mm). Scape de la femelle (fig. 13) non élargi (largeur: 0,055-0,06 mm). Edéage (long. 0,35$0,39 \mathrm{~mm}$ ) avec les deux apophyses grêles du sac interne de même longueur (cf. KarAmAN, 1957: 203, fig. 72); paramères ornés sur la face ventrale d'une rangée de 3 ou 4 petites dents, plus rarement 5, oblique par rapport à l'axe de symétrie de l'édéage (fig. 14 et 15).

Croatie, Slovénie, Carinthie, Styrie, Frioul, Vénétie et province de Brescia. 
B. longulus inflatus $n$. ssp. Carénule médiane du vertex égale sur toute sa longueur, ornée parfois en avant d'une saillie arrondie très légère. Scape du mâle (fig. 16) fortement renflé (largeur: 0,10-0,105 $\mathrm{mm}$ ). Scape de la femelle (fig. 17) un peu élargi (largeur: 0,065-0,07 mm). Edéage (long. 0,37-0,39 $\mathrm{mm}$ ) avec les deux apophyses grêles du sac interne nettement inégales; paramères ornés sur la face ventrale d'une rangée de 5 ou 6 petites dents, plus rarement 4, parallèle à l'axe de symétrie de l'édéage (fig. 18 et 19).

Italie. Piémont. Prov. Novara: Monte Fenera près de Borgosesia, 6 ô 27 o (Focarile) (holotype $\hat{0}$, Mus. Genève; paratypes: Coll. A. Focarile et Mus. Genève) en octobre 1981, dans la litière de noisetiers entre 500 et 800 m d'altitude; Varallo Sesia, 1 ô (Dodero) (paratype, Mus. Frey, Tutzing); Rima, 7 ㅇ 3 우 (Breit) (paratypes, Mus. Frey, Tutzing et Mus. Genève).

\section{Bryaxis reissi (Mach.)}

Bryaxis reissi Machulka [1933: 3; type đ̃: Cima Tombea/Italie (! Mus. Innsbruck)] a enfin été retrouvé, cinquante ans après sa découverte! Sa description est exacte, mais

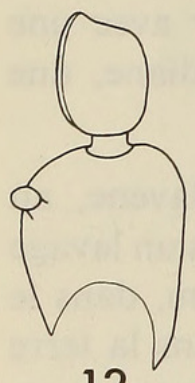

12
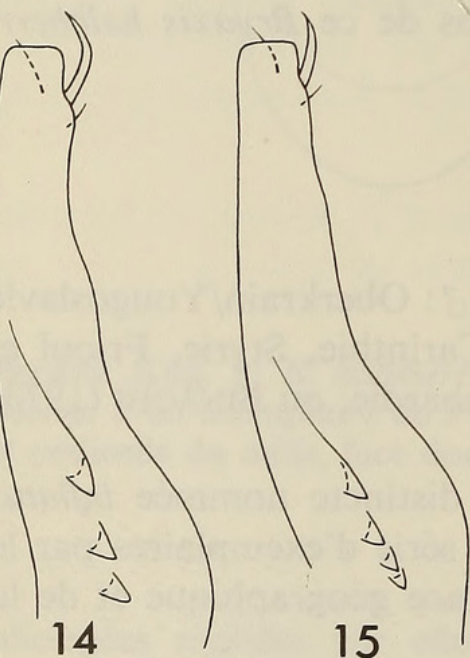

15

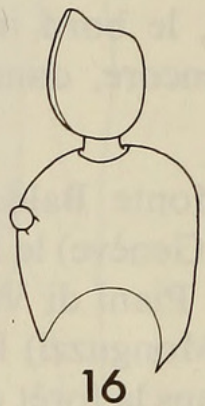

13

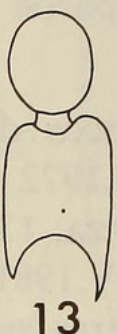

16
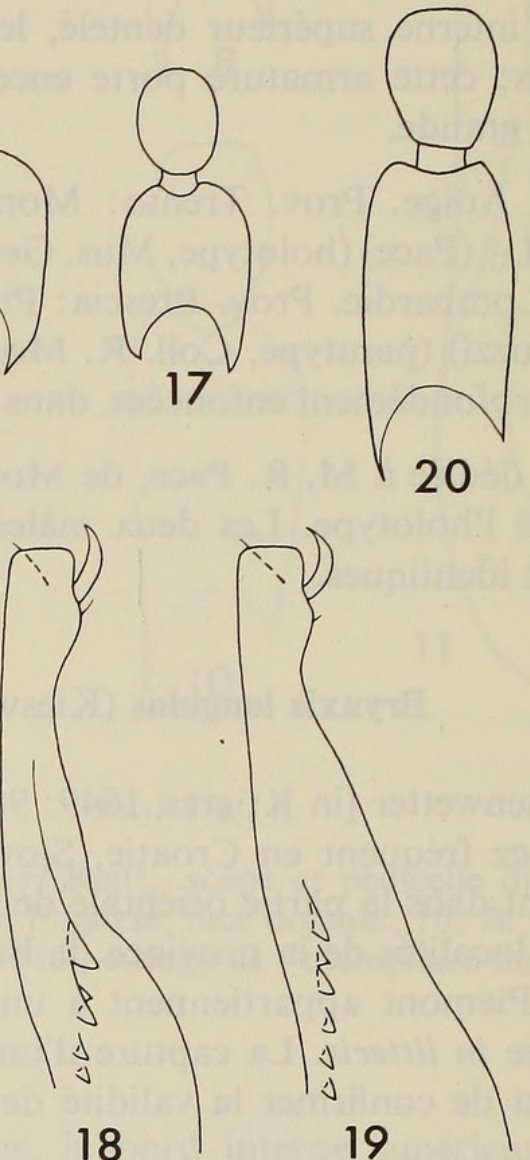

19

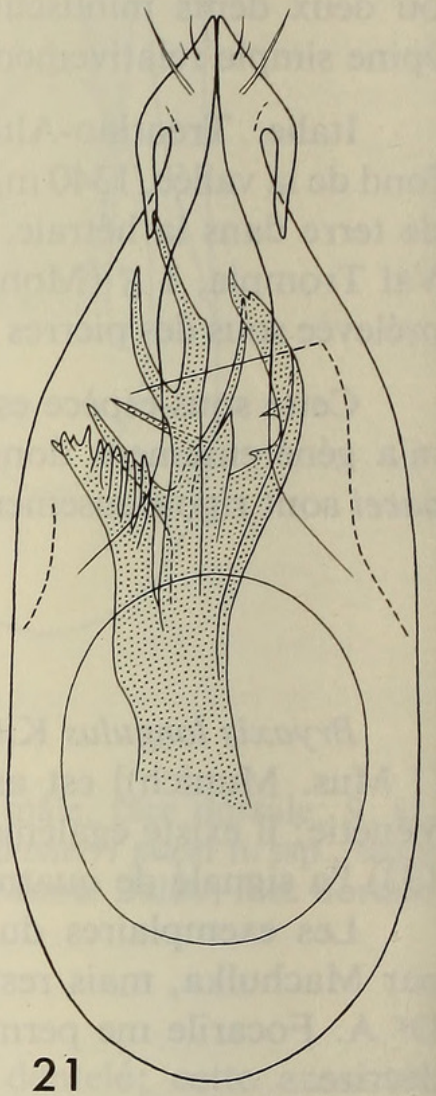

21

FiG. 12 à 21.

Bryaxis Kug. 12: B. longulus longulus Kiesw., scape et pédicelle du mâle, face dorsale; 13: id., scape et pédicelle de la femelle, face dorsale; 14 :id., paramère droit d'un exemplaire des environs de Bleiburg, face ventrale; 15: id., paramère droit d'un exemplaire de Musi, face ventrale; 16: B. longulus inflatus $\mathrm{n}$. ssp., scape et pédicelle du mâle, face dorsale; 17 :id., scape et pédicelle de la femelle, face dorsale; 18: id., paramère droit d'un exemplaire du Monte Fenera, face ventrale; 19: id., paramère droit d'un exemplaire de Rima; 20: B. reissi Mach. du M. Pizzocolo, scape et pédicelle du mâle, face dorsale; 21 : id., édéage, face dorsale. 
je profite cependant de la compléter puisque j'ai sous les yeux les deux types de la Cima Tombea (Collection O. Reiss, Innsbruck) et les deux mâles du M. Pizzocolo (Collection R. Monguzzi, Milan et Mus. Genève).

Long. 1,6 (Cima Tombea) à 1,7 mm (M. Pizzocolo). Yeux peu développés, formés chacun de 4 à 5 ommatidies chez le mâle, de 2 à 3 chez la femelle. Palpes maxillaires particulièrement courts, le dernier article un peu plus long chez la femelle $(0,26 / 0,10 \mathrm{~mm})$ que chez le mâle $(0,23 / 0,10 \mathrm{~mm})$. Antennes relativement longues $(0,78 \mathrm{~mm}$ pour les ex. de la Cima Tombea, 0,84-0,85 pour ceux du M. Pizzocolo), semblables dans les deux sexes; scape $(0,17-0,18 / 0,07-0,08 \mathrm{~mm})$ et pédicelle $(0,075-0,08 / 0,055-0,06 \mathrm{~mm})$ (fig. 20) simples chez le mâle. Fémurs peu renflés chez le mâle de la Cima Tombea (largeur fémurs I et III: 0,11 et $0,12 \mathrm{~mm}$ ), distinctement plus renflés chez les mâles du M. Pizzocolo (largeur fémurs I et III: 0,12 et $0,13 \mathrm{~mm}$ ).

Edéage (fig. 21). Long. 0,37 (Cima Tombea) à 0,40 mm (M. Pizzocolo). Paramères armés un peu avant l'apex d'une dent latérale grêle assez longue. Armature du sac interne asymétrique, très complexe, semblable pour les deux provenances.

Italie. Lombardie. Prov. Brescia: Cima Tombea, 1 ô 1 † (Reiss) (types, Mus. Innsbruck); M. Pizzocolo, 1200 m, 2 ô (Monguzzi) le 15.V.1982, dans la terre prélevée sous des pierres profondément enfoncées dans la forêt de hêtres, sapins et ormes.

\section{Bryaxis sorinensis (Stolz)}

Je dois faire tomber dans la synonymie du Bryaxis sorinensis Stolz [1917: 28; type o : Val Sorina/Italie (! Mus. Vienne)] mon Bryaxis bergamascus breiti Besuchet [1980: 625; type $\sigma^{\hat{*}}$ : Mte Guglielmo/Italie (! Mus. Frey, Tutzing)]. Les édéages sont identiques, de même que les scapes et l'ensemble des caractères morphologiques. Les antennes du type de $B$. sorinensis sont cependant légèrement plus allongées: pédicelle un peu plus long que large, article 3 nettement plus long que large, 4 et 5 aussi longs que larges (chez breiti: pédicelle du mâle presque sphérique, article 3 un peu plus long que large, 4 et 5 un peu plus larges que longs).

Bryaxis bergamascus sorinensis Stolz est ainsi connu de deux localités bien distinctes.

Italie. Lombardie. Prov. Brescia: Mte Guglielmo, 2 o 3 \& (Breit). Trentino-Alto Adige. Prov. Trento: Val Sorina près de Storo, 1 đ̂ (Pinker) le 20.VI.1907, dans un tamisage de feuilles mortes de hêtre.

\section{BIBLIOGRAPHIE}

Besuchet, C. 1980. Contribution à l'étude des Coléoptères Psélaphides d'Italie et du Tessin. Revue suisse Zool. 87: 611-635.

BinAGHI, G. 1973. Contributo allo studio degli Pselafidi delle Prealpi Lombarde con particolare riguardo ai Bythinini. Memorie Soc. ent. ital. 52: 99-139.

DaffNer, H. 1982. Eine neue Art der Gattung Bryaxis Kugelann aus Mitteleuropa. NachrBl. bayer. Ent. 31: 112-115.

Dodero, A. 1900. Materiali per lo studio dei Coleotteri italiani con descrizioni di nuove specie. Annali Mus. civ. Stor. nat. Giacomo Doria, Ser. 2a, XX: 400-419.

Ganglbauer, L. 1895. Die Käfer von Mitteleuropa. II. Wien, 881 p.

Karaman, Z. 1957. Die balkanischen Bythininen (Col. Pselaphidae). Ihre Systematik, Zoogeographie und Phylogenie. Biol. Glasnik, Zagreb 10: 161-208. 
KÜSTER, H. C. 1849. Die Käfer Europas. XVI. Nürnberg, 100 p.

MachulKa, V. 1933. Ein neuer Bythinus von Norditalien. Ent. NachrBl., Troppau 7: 3-5.

ReitTeR, E. 1885. Neue Coleopteren aus Europa und den angrenzenden Ländern, mit Bemerkungen über bekannte Arten. Dt. ent. Z. 29: 353-402.

Stolz, H. 1917. Neue Bythinusarten aus Oberitalien und Südtirol. Wien. ent. Ztg. 36: 19-31. 


\section{$2 \mathrm{BHL}$ Biodiversity Heritage Library}

1983. "Bryaxis nouveaux ou meconnus du nord de I'Italie (Coleoptera: Pselaphidae)." Revue suisse de zoologie 90, 769-780. https://doi.org/10.5962/bhl.part.82013.

View This Item Online: https://www.biodiversitylibrary.org/item/128827

DOI: https://doi.org/10.5962/bhl.part.82013

Permalink: https://www.biodiversitylibrary.org/partpdf/82013

\section{Holding Institution}

Smithsonian Libraries

\section{Sponsored by}

Biodiversity Heritage Library

\section{Copyright \& Reuse}

Copyright Status: In Copyright. Digitized with the permission of the rights holder.

Rights Holder: Muséum d'histoire naturelle - Ville de Genève License: http://creativecommons.org/licenses/by-nc-sa/3.0/

Rights: https://www.biodiversitylibrary.org/permissions/

This document was created from content at the Biodiversity Heritage Library, the world's largest open access digital library for biodiversity literature and archives. Visit BHL at https://www.biodiversitylibrary.org. 